\title{
Molecular Dynamics Study of Fluid Solid Interfacial Slip and Its Effect on Aerodynamic Drag
}

\author{
Asef Mohammed ${ }^{\mathrm{a}}$ and Jeetu S. Babu \\ Department of Mechanical Engineering, Amrita School of Engineering, Amritapuri, \\ Amrita Vishwa Vidyapeetham, Amrita University, India
}

\begin{abstract}
Interfacial hydrodynamic slippage is controlled by two factors say physical structure and chemical composition. Various studies have been conducted experimentally which try to connect the physical structure of the surface and its chemical property on the interfacial wettability. One such example is the Tunable wettability in surface-modified ZnO-based hierarchical nanostructures [2]. In which vertically aligned Nanoneedles and Nanonails were employed as a platform to determine the effect of surface structure. According to which a variation in static contact angles were observed as the cap size the nanonails constantly increased. Starting with a contact angle of $104^{\circ}$ the contact angle first increases and then decreases, which means that the slip length first increases and then decreases. The increase in slip length reduces the drag, which has immense application in the aerodynamic field. This paper investigates the relation between the chemical wettability and aerodynamic drag by performing MD simulations of couette flow with varying fluid-surface interaction.
\end{abstract}

\section{Introduction}

The fluid drag force acting on a moving solid body in the direction of the free stream flow of the fluid is termed as aerodynamic drag in aerodynamics. The forces due to pressure distribution over the surface result in drag. Reducing the drag plays a major role in increasing the stability of a moving body, It becomes significant when aerospace is the subject under consideration. You and Moin showed that the slip velocity present in hydrophobic surfaces could result in a significant drag reduction [1]. It could be concluded from their work that higher the slip length, higher will be the reduction in drag and rms lift. Since it is clear that slip and drag are directly related, in this work we investigate methods to manipulate slip in order to reduce drag with the help of surface modifications.

Various studies have been conducted experimentally as well as numerically which try to connect the physical structure of the surface and its chemical property on the interfacial wettability. One such example is the tunable wettability in surface modified $\mathrm{ZnO}$-based hierarchical nanostructures by Li et. al[2]. In which vertically aligned nanoneedles and nanonails are used to study the deviation of surface structure, according to which a variation in static contact angles were observed as the size of the nanonail cap constantly increased. Starting with a contact angle of $104^{0}$ the contact angle first increases and then decreases, which means that the slip length first increases and then decreases. In a numerical work done by Bakli and Misra [3] on the comparability of chemical composition and

\footnotetext{
${ }^{a}$ Corresponding author: asefmohammed3@gmail.com
} 
roughness of Nano channels in varying slippage of fluid at the interface through molecular dynamics (MD) simulations, by developing a typical water model the slippage of fluid through smooth and rough surfaces were analysed. The authors proposed a mathematical relation between static contact angle, surface roughness and slip length by determining the contact angles and slip length at different conditions. In a similar work done by Bakli and Chakraborty [4] they have studied the transition of slippery to sticky hydrophobic nano channels. They showed that a simple manipulation of the ionic and molecular positioning at the wall- fluid interface can be used to tune the actual wettability of the nano fluidic substrate. In this work they have opened up a new platform for studying the slip-stick behaviour by making the classification of hydrophobic and hydrophilic surfaces slightly imprecise, which they made possible by using colloidal and ionic inclusions in the fluid which considerably affected the dynamic contact angle.

The objective of this paper is to reduce drag by relating it with slip by applying surface modifications. In order to support the experimental work done by You and Moin [1] we have tried to mimic the idea of nano needles and nails by creating cylindrical and square shaped surface asperities by varying the contact angle. Further studies are also done by varying the wall-fluid interaction in order to change the contact angle and hence to manipulate slip length.

\section{Methodology}

This work is done using MD simulation [5]. MD can be explained as a computational tool where positions, velocities and orientation of molecules and atoms evolve over time. It is one of the most widely used atomistic simulation method. It can be considered as a computational microscope, where the behaviour of atoms and molecules are observed using a computational technique instead of using a conventional microscope. With the help of MD we can get atomistic insight into molecular structure and kinetics. It is a deterministic method, given a preliminary set of position and velocities the following time evolution is totally calculated so that the entire trajectory of the atoms could be obtained. MD follows laws of classical mechanics most notably Newton's law: $F=M \times a$, where $F$ is the net force acting on the atom, $M$ is the mass of the atom and $a$ is the acceleration. The total force acting on the atom can be calculated from the interaction between the atom and its neighbouring atoms. This can be modelled using an interatomic potential function. The force acting on the atom is defined as the first derivative of the interatomic potential function with respect to position. Once the total force acting on the atom is obtained, the acceleration can be calculated and from the acceleration of the atom the positions and velocities can be obtained by time integration. So, in MD we are actually solving the Newton's law for obtaining the trajectory of the atoms. From the atom trajectory the properties of the system can be calculated with the help of statistical mechanics relation.

Hence, Running a simulation in MD can be summarised as follows

1. Set an initial system configuration $[\vec{r}(\mathrm{t}=0), \vec{v}(\mathrm{t}=0)]$

2. Get an interaction potential for the system $\mathrm{v}(\vec{r})$

3. Find a way to integrate $F=M \times a$ and get new forces $\mathrm{F}$

4. Solve the equation of motion numerically over a short step $\Delta t$

5. Get the new positions and velocities over the step $\Delta t$

6. Continue step 1-5 till $\mathrm{t}>t_{\max }$

7. Calculate the results and stop 
The basic flowchart for MD simulation is shown in Fig. 1

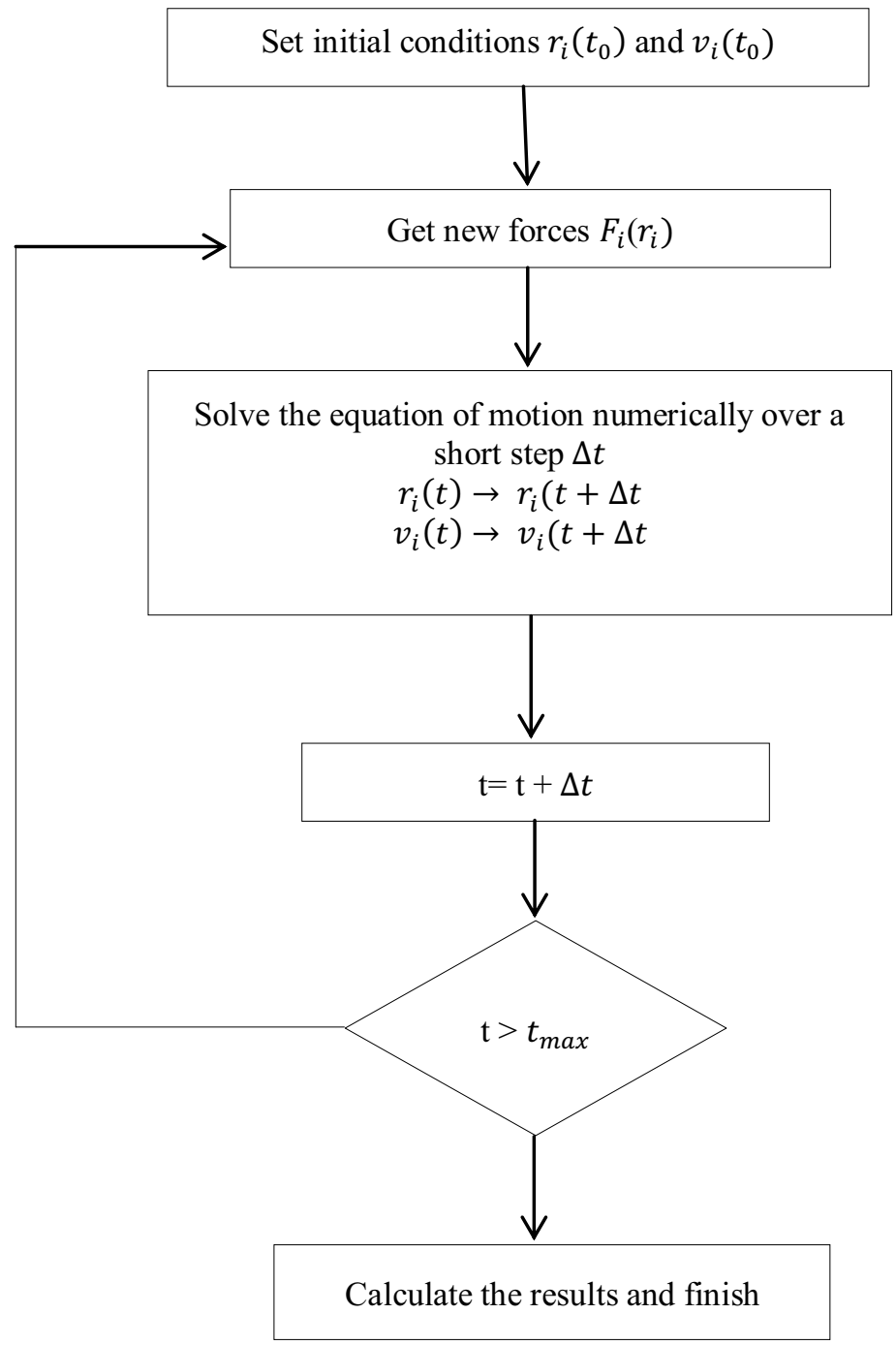

Figure 1. MD simulation flow chart

The MD simulation is implemented using LAMMPS open source library package [6,7] and the visualisation of the same is done using OVITO [8] which is also an open source software. The simulation system consists of a channel of height $1 \mathrm{~nm}$ bounded by rigid solid walls in close pack face centred cubic (FCC) structure. Argon and platinum atoms constitute the fluid and wall respectively. The complete dimension of the system is $8 \times 3.2 \times 8 \mathrm{~nm}$ as shown in Fig. 2. The system is designed for Couette flow so that the upper plate moves with a small velocity $\mathrm{V}$ and the lower plate is kept stationary. 


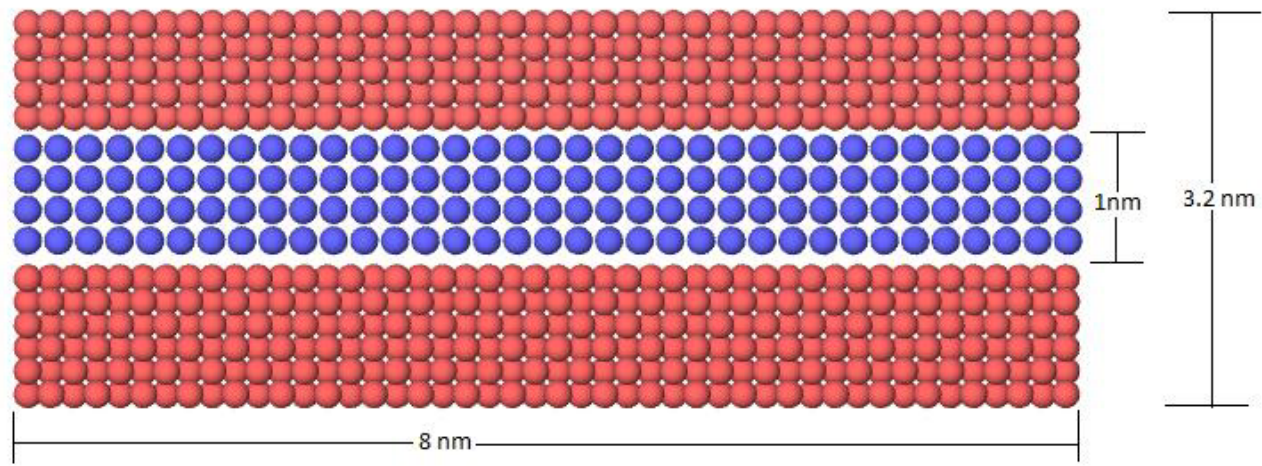

Figure 2. Couette flow simulation system in two dimensional view

Lenard Jones (LJ) interaction potential is used to model the wall-wall, fluid-fluid and wall-fluid interactions.

$$
\phi L J(r)=4 \varepsilon\left[\left(\frac{\sigma}{r}\right)^{12}-\left(\frac{\sigma}{r}\right)^{6}\right]
$$

The interaction parameter between the atoms of the wall and fluid are as shown in Table- 1 and $\varepsilon_{w f}$ is varied to change the wall fluid interaction and hence the contact angle. The second part of the work is done by creating square and cylindrical surface asperity in the lower wall so us to vary the contact angle. Fig. 3 shows the simulation of couette flow with surface asperity. The complete dimension of the system is $8 \times 3.2 \times 8 \mathrm{~nm}$.

Table 1. Interaction parameters

\begin{tabular}{|l|l|l|}
\hline & $\boldsymbol{\varepsilon}(\mathbf{k c a l} / \mathbf{m o l e})$ & $\boldsymbol{\sigma}\left(\mathbf{A}^{\mathbf{0}}\right)$ \\
\hline Wall-wall & 0.120183 & 2.475 \\
\hline Fluid-fluid & 0.238 & 3.04 \\
\hline Wall-fluid & \multicolumn{1}{|c|}{-} & 2.7575 \\
\hline
\end{tabular}
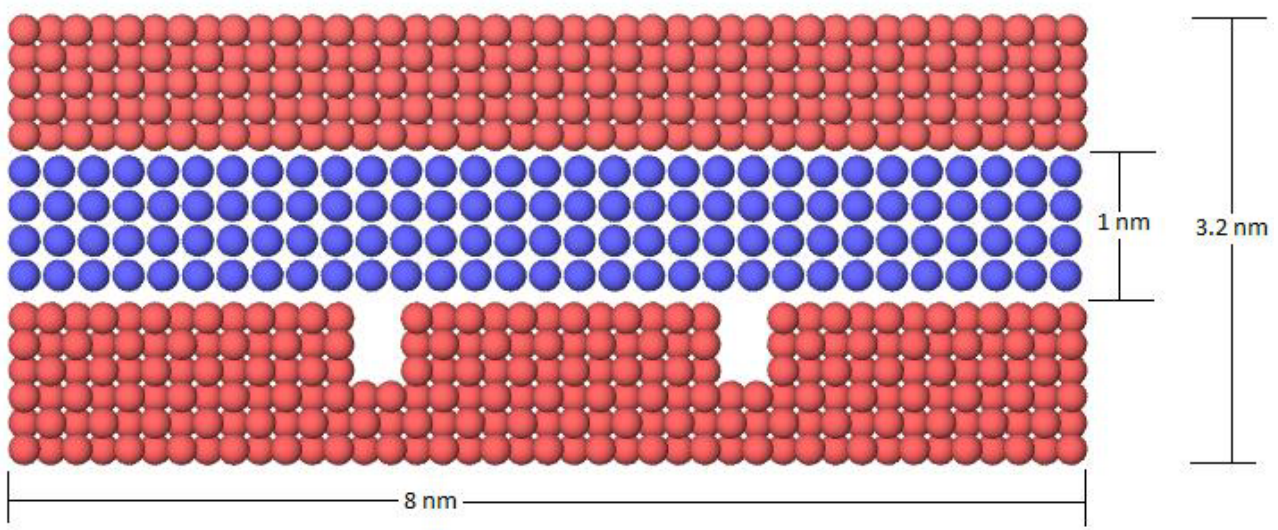

Figure 3. Couette flow simulation system with surface asperity in two dimensional view

Extrapolating the velocity profile obtained through MD simulation of Couette flow to meet the velocity of the wall will give the slip length. The numerical calculation of slip length was done by 
using the formula $V_{S}=L_{s} \times \dot{\gamma}$, where $V_{s}$ is the slip velocity; $L_{s}$ is the slip length and $\dot{\gamma}$ is the slope. The total energy exerted by the wall on the fluid slip layer is also calculated in every case. The relation between total energy and slip length is also studied.

\section{Results and Discussions}

All the values obtained are represented with respect to the values obtained for $\varepsilon_{w f}=0.169 \mathrm{kcal} / \mathrm{mole}$. First part of the work was done by varying the wall fluid interaction in order to vary the contact angle and the variation of slip length observed is as shown in the Fig. 4 and the variation of total energy observed is as shown in Fig. 5

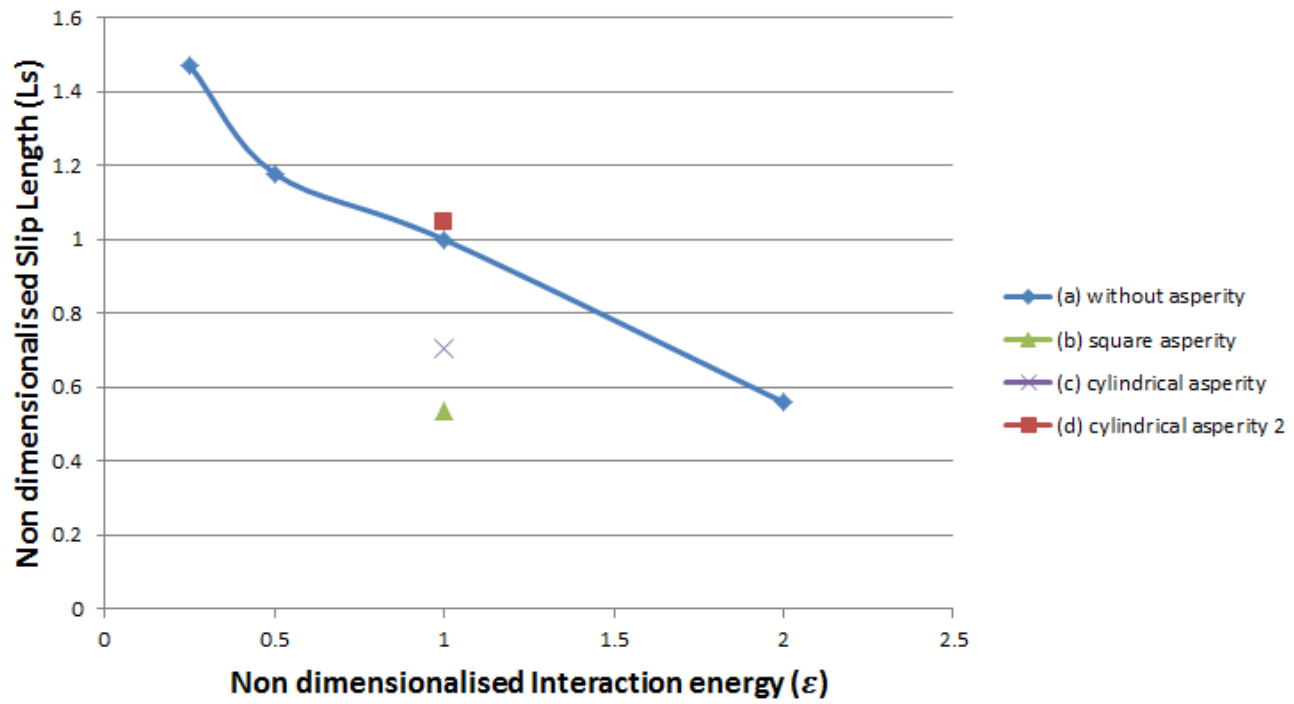

Figure 4. Variation of slip length with respect to changes in the wall-fluid interaction energy (»), Changes in shape of the surface asperities [square $(\Delta)$ and cylinder $(\times, \varpi)$ ] and also changes in the surface area of the asperity $[\times, \square]$.

As shown in Fig. 5 when the wall-fluid interactions were reduced, the total energy exerted on the fluid atoms by the wall was found to reduce as well, as a result of which the fluid transformed from philic to phobic hence, the slip length increased. The increase in slip length with reduction in wallfluid interaction $(\varepsilon)$ is shown in Fig. 4. The result obtained when surface asperity was created in order to change the contact angle is also shown in the Fig. 4.

Figure 4 shows the variation of slip length with respect to flat plate, cylindrical asperity and square asperity for a particular value of $\varepsilon$. The slip length is found to reduce from what was obtained from flat plate, when asperities were created. This can be explained due to the change in contact angle such that when the contact angle increases the slip length reduces. A further reduction in slip length was found when the asperity shape was changed from cylindrical to square by assuring the same surface area. This effect can also be explained as a result of change in contact angle which can be supported by the work done by Li et. $\mathrm{Al}[2]$. 


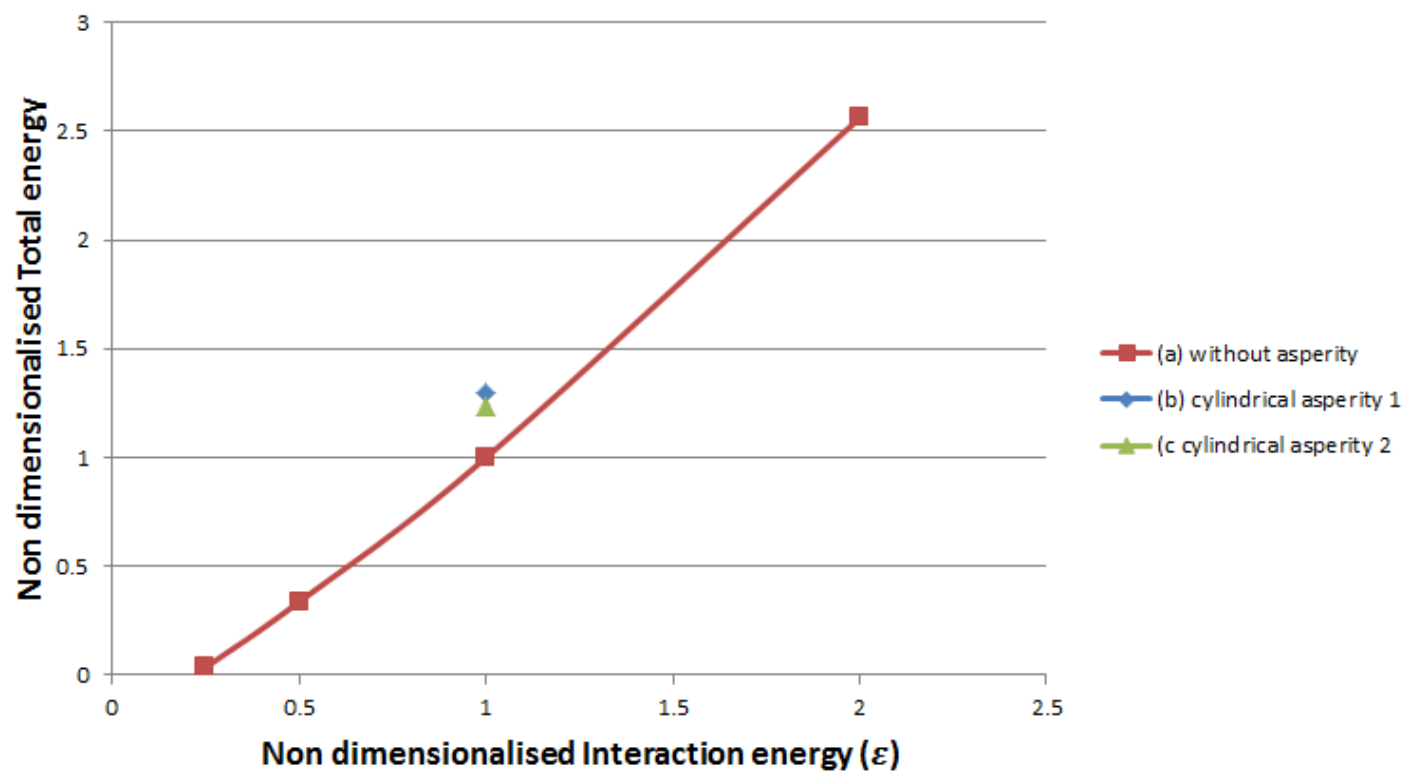

Figure 5. Variation of total energy with respect to change in the wall fluid interaction energy ( $\square)$ and also change in the surface area of the asperity $(\diamond, \triangle)$.

In an extended analysis done by reducing the surface area of the cylindrical asperity, a further reduction in total energy and an increase in slip length was found. Due to the reduction in contact angle the total energy exerted on the fluid by the wall will increase as shown in Fig. 5 and as a result due to change in phobic to philic nature the slip length will reduce as shown in the Fig. 4.

\section{Conclusion}

Through MD simulation of Couette flow, the slippage of fluid through hydrophobic surfaces were studied. By measuring slip length for various conditions of varying contact angles the manipulation of slip was made possible. Varying the wall-fluid interaction energy as well as creating surface asperity were tried in order to change the contact angle. The manipulation of slip length can considerably effect the drag which could be used for aerodynamic applications.

\section{References}

1. D. You, P. Moin, Physics of Fluids 19, 081701 (2007)

2. G. Li, T. Chen, B. Yan, Y. Ma, Z. Zhang, T. You, Z. Shen, H. Chen, T. Wu, Appl. Phys. Lett. 92, 173104 (2008)

3. C. Bakli, A. Misra, Physics.flue-dyn, arXiv :1605.02479 (2016)

4. C. Bakli, S. Chakraborty, Nano Lett. 15, 7497-502 (2015)

5. C. Rapaport, The Art of Molecular Dynamics Simulation, Cambridge university press New York, 2004

6. S. Plimpton, Fast Parallel Algorithms for Short-Range Molecular Dynamics, J Comp Phys, 117, 19 (1995).

7. http://lammps.sandia.gov

8. A. Stukowski, Visualization and analysis of atomistic simulation data with OVITO - the Open Visualization Tool Modelling Simul. Mater. Sci. Eng. 18, 015012 (2010) 